\title{
A dynamical model for the dusty ring in the Coalsack
}

\author{
P. Hennebelle ${ }^{1}$, A. P. Whitworth ${ }^{2}$, and S. P. Goodwin ${ }^{3}$
}

\author{
${ }^{1}$ École Normale Supérieure, Laboratoire de Radioastronomie Millimétrique, 24 rue Lhomond, 75231 Paris Cedex 05, France \\ e-mail: Patrick. Hennebelle@ens . fr \\ 2 School of Physics \& Astronomy, Cardiff University, 5 The Parade, Cardiff CF24 3YB, Wales, UK \\ e-mail: Anthony. Whitworth@astro.cf.ac.uk \\ 3 Department of Physics \& Astronomy, University of Sheffield, Hicks Building, Housfield Road, Sheffield S3 7RH, UK \\ e-mail: S.Goodwin@sheffield.ac.uk
}

Received 18 July 2005 / Accepted 2 February 2006

\begin{abstract}
Lada et al. recently presented a detailed near-infrared extinction map of Globule G2 in the Coalsack molecular cloud complex, showing that this starless core has a well-defined central extinction minimum. We propose a model for G2 in which a rapid increase in external pressure is driving an approximately symmetric compression wave into the core. The rapid increase in external pressure could arise because the core has recently been assimilated by the Coalsack cloud complex, or because the Coalsack has recently been created by two large-scale converging flows. The resulting compression wave has not yet converged on the centre of the core, so there is a central rarefaction. The compression wave has increased the density in the swept-up gas by about a factor of ten, and accelerated it inwards to speeds of order $0.4 \mathrm{~km} \mathrm{~s}^{-1}$. It is shown that even a low initial level of turbulence completely destroys the coherence of the ring, as seen in projection. In the scenario of strong external compression that we are proposing this implies that the initial turbulent energy in the globule was low, $E_{\mathrm{TURB}} /\left|E_{\mathrm{GRAV}}\right| \leq 2 \%$. Protostar formation should occur in about 40000 years.
\end{abstract}

Key words. ISM: clouds - stars: formation - hydrodynamics - instabilities - shock waves

\section{Introduction}

In the current paradigm for star formation, single stars, binary and multiple stars, and even small-N clusters, are presumed to form following the collapse and fragmentation of a dense molecular core (e.g. André et al. 2000). However, many details of this paradigm remain to be understood, in particular the processes which form dense molecular cores in the first place, and thereby determine the initial conditions for collapse and fragmentation.

In an attempt to understand these processes, Lada and colleagues have explored the structures of several cores, using the NICE technique (Alves et al. 1998). These include B68 (Alves et al. (2001), where there is evidence that the core is oscillating, in accordance with the predictions of Matsumoto \& Hanawa (2003) and Hennebelle (2003), and B335 (Harvey et al. 2001), where the density profile suggests that the core has already become unstable against collapse. In a more recent application of the NICE technique, Lada et al. (2004) have mapped Tapia's Globule 2 in the Coalsack (Tapia 1973).

The Coalsack is an extended cloud complex at a distance $D \sim 150$ to 175 pc (Cambresy 1999; Rodgers 1960). If we adopt the smaller distance, the angular extent of the complex $\left(\sim 6^{\circ}\right)$ corresponds to a linear extent of $\sim 15 \mathrm{pc}$, and the mass of molecular gas is $\sim 3000 M_{\odot}$ (Nyman et al. 1989).
The highly structured molecular gas mapped by Nyman et al. is probably accompanied by an envelope and/or infill of somewhat more diffuse HI, providing shielding from the ambient $\mathrm{H}_{2}$-dissociating UV radiation field. This HI would be very hard to detect unambiguously, since the systemic velocity of the Coalsack is very low $\left(\sim-5\right.$ to $\left.0 \mathrm{~km} \mathrm{~s}^{-1}\right)$ and there would therefore be confusion with local $21 \mathrm{~cm}$ emitting gas.

Despite its molecular content, and therefore presumably relatively high density, the Coalsack cloud complex shows no unambiguous evidence for ongoing star formation. There are no flare stars (Weaver 1973); no T Tauri stars (Weaver 1974a,b); no $\mathrm{H} \alpha$ emission stars (Schwartz 1977); and no IRAS sources associated with either the extinction peaks or CO emission peaks (Nyman et al. 1989). Reipurth detected a single HH object, R10, in the direction of the Coalsack, and Eaton et al. (1990) showed it to be a bipolar nebula with a heavily obscured illuminating star. However, Kato et al. (1999) question whether R10 is really associated with the Coalsack, and it is certainly a long way from Globule 2 (hereafter G2).

It is therefore possible that $\mathrm{G} 2$, which appears to be the densest core in the Coalsack, is in an early stage of condensing to form a protostar (or protostars) and, if so, its detailed structure could contain important clues about how cores form. To this end, Lada et al. (2004) have obtained deep near-IR 
photometry of 24000 stars seen through G2, and have used this data to construct an extinction map of the core with unprecedented resolution.

The most remarkable feature of this map is that the core has a local extinction minimum in the middle, and the maximum extinction is in a ring-like structure around this minimum. Lada et al. discuss some possible explanations for this structure, under the general headings: "flattened geometry and magnetic fields" and "spherical geometry: a core in transition". We here present a detailed model under the second heading, in which G2 is a core responding to a sudden increase in external pressure as investigated in Hennebelle et al. (2003, 2004). This increase in external pressure is driving a compression wave into the cloud, producing a dense shell which - seen in projection - is visible as a ring of enhanced extinction. The broad ${ }^{12} \mathrm{C}^{18} \mathrm{O}(J=2 \rightarrow 1)$ emission line observed by Lada et al. along a single line of sight through the core is then attributable to the extra trans-sonic velocity generated by the inward-propagating compression wave.

In Sect. 2 we describe briefly the code used to simulate this model, and the initial conditions adopted. In Sect. 3 we present the results obtained and compare them to the observations; it should be emphasised at the outset that we do not perform an extensive parameter search to obtain the best fit to the observational data, since this does not, at the present time, seem justified by the scope of the observations. In Sect. 4 we discuss the results, attempt to justify key aspects of the model, and summarise our main conclusions.

\section{Numerical method and initial conditions}

The simulations were performed using the SPH code DRAGON, with $\sim 500000$ particles. This code invokes an octtree to find neighbours and calculate gravitational accelerations. The particle smoothing lengths are adjusted so that each particle has $\mathcal{N}_{\text {NEIB }}=50 \pm 5$ neighbours. A second-order Runge-Kutta integration scheme is used, with multiple particle time-steps. Other details of the code are given in Goodwin et al. (2004).

In the first instance we present a simulation with zero turbulence, since this seems to capture well the azimuthally averaged features of the ring-like structure reported by Lada et al. (2005). The initial conditions for this simulation are a truncated Bonnor-Ebert sphere with boundary at radius $R_{\mathrm{B}}=$ $3 a_{0} /\left(4 \pi G \rho_{\mathrm{C}}\right)^{1 / 2}$, where $a_{0}$ is the isothermal sound speed and $\rho_{\mathrm{C}}$ is the central density. We set $a_{0}=0.2 \mathrm{~km} \mathrm{~s}^{-1}$ (corresponding to molecular gas at $T_{0}=10 \mathrm{~K}$ ) and $\rho_{\mathrm{C}}=0.2 \times 10^{-20} \mathrm{~g} \mathrm{~cm}^{-3}$ (corresponding to $n_{\mathrm{C}}=0.5 \times 10^{3} \mathrm{H}_{2} \mathrm{~cm}^{-3}$ ). This gives a core radius $R_{\mathrm{B}}=0.4 \mathrm{pc}$ and a total core mass $M_{\mathrm{TOTAL}}=6.5 M_{\odot}$. The cloud is in stable equilibrium with external pressure $P_{\mathrm{EXT}}=P_{0}=$ $0.22 \times 10^{-12} \mathrm{erg} \mathrm{cm}^{-3} \equiv 0.16 \times 10^{4} k_{\mathrm{B}} \mathrm{cm}^{-3} \mathrm{~K}$. The initial conditions are set up with a random but settled (i.e. non-crystalline) distribution of particles in detailed hydrostatic equilibrium, and the subsequent evolution is isothermal (i.e. $P=a_{0}^{2} \rho$, with uniform and constant $a_{0}$ ). At $t=0$, we increase $P_{\mathrm{EXT}}$ at a constant
Table 1. Parameters of the initial turbulence in the different simulations.

\begin{tabular}{cccc}
\hline \hline Simulation & $\alpha_{\text {TURB }}$ & $n$ & Figs. \\
\hline 1 & 0 & - & 1 and 2 \\
2 & 0.01 & $5 / 3$ & 3 \\
3 & 0.02 & $5 / 3$ & 4 \\
4 & 0.02 & 0 & 5 \\
\hline
\end{tabular}

rate, by a factor of 10 , over a thirtieth of a crossing time, i.e.

$$
P_{\mathrm{EXT}}=\left\{\begin{array}{lr}
P_{0}, & t \leq 0 \\
\left(1+270 t / t_{\mathrm{SC}}\right) P_{0}, & 0<t \leq t_{\mathrm{SC}} / 30 \\
10 P_{0}, & t>t_{\mathrm{SC}} / 30
\end{array}\right.
$$

The sound-crossing time is given by $t_{\mathrm{SC}}=R_{\mathrm{B}} / a_{0}=2 \mathrm{Myr}$, and so the new increased external pressure is reached after $\sim 0.07$ Myr.

We then explore how the evolution of the core is modified if it has a modest level of internal turbulence. This is motivated by the fact that the ring-like structure observed by Lada et al. presents significant departures from an ideal axisymmetric ring. We therefore explore what level of turbulence is compatible with the observed ring pattern.

The turbulence is characterised by two parameters: (i) the ratio of turbulent energy, $E_{\mathrm{TURB}}$, to self-gravitational potential energy, $E_{\mathrm{GRAV}}$, i.e. $\alpha_{\mathrm{TURB}}=E_{\mathrm{TURB}} / E_{\mathrm{GRAV}}$; and (ii) the exponent $n$ in the turbulent power spectrum, $P(k) \propto k^{-n}$. The turbulent parameters in the different simulations are given in Table 1.

\section{Simulation results}

\subsection{Spherical case}

Figure 1 shows the profiles of volume-density, $n(r)$, inflow velocity, $u(r)$, and column-density, $N(b)$, generated by the nonturbulent simulation (simulation 1 in Table 2 ) at three times, $t=1.170,1.183$, and $1.198 \mathrm{Myr}$ (i.e. well after the external pressure has reached its new increased value, $10 P_{0}$ ). Here $r$ is distance from the centre of the core and $b$ is the impact parameter of the line of sight relative to the centre of the core. The density in the swept-up shell has been increased from $n \sim 0.3 \times 10^{3} \mathrm{~cm}^{-3}$ to $n \sim 0.6 \times 10^{4} \mathrm{~cm}^{-3}$, and the swept-up shell is travelling inwards at $u \sim 0.4 \mathrm{~km} \mathrm{~s}^{-1}$ (i.e. Mach-2).

If we adopt the relation $N=0.2 \times 10^{22} \mathrm{~cm}^{-2} \times A_{\mathrm{V}}$ (where $N$ is the column-density of hydrogen nuclei in all forms, and $A_{\mathrm{V}}$ is the visual extinction in magnitudes), we can convert the azimuthally averaged extinction profile obtained by Lada et al. (2004) from their near-IR measurements through G2 (their Fig. 3) into a column-density profile. The result of this conversion is shown as a dashed line in the righthand panels of Fig. 1. We see that the observed column-density profile is fitted best by the simulated column-density profile at $t=1.183 \mathrm{Myr}$. The simulated profile has a minimum of $N \sim 0.16 \times 10^{23} \mathrm{~cm}^{-2}$ in the centre, and a maximum of $N \sim 0.24 \times 10^{23} \mathrm{~cm}^{-2}$ at radii $r \sim 0.04 \mathrm{pc}$, close to the maximum of the observed profile. We note that, with a spherically symmetric model, it requires a very 

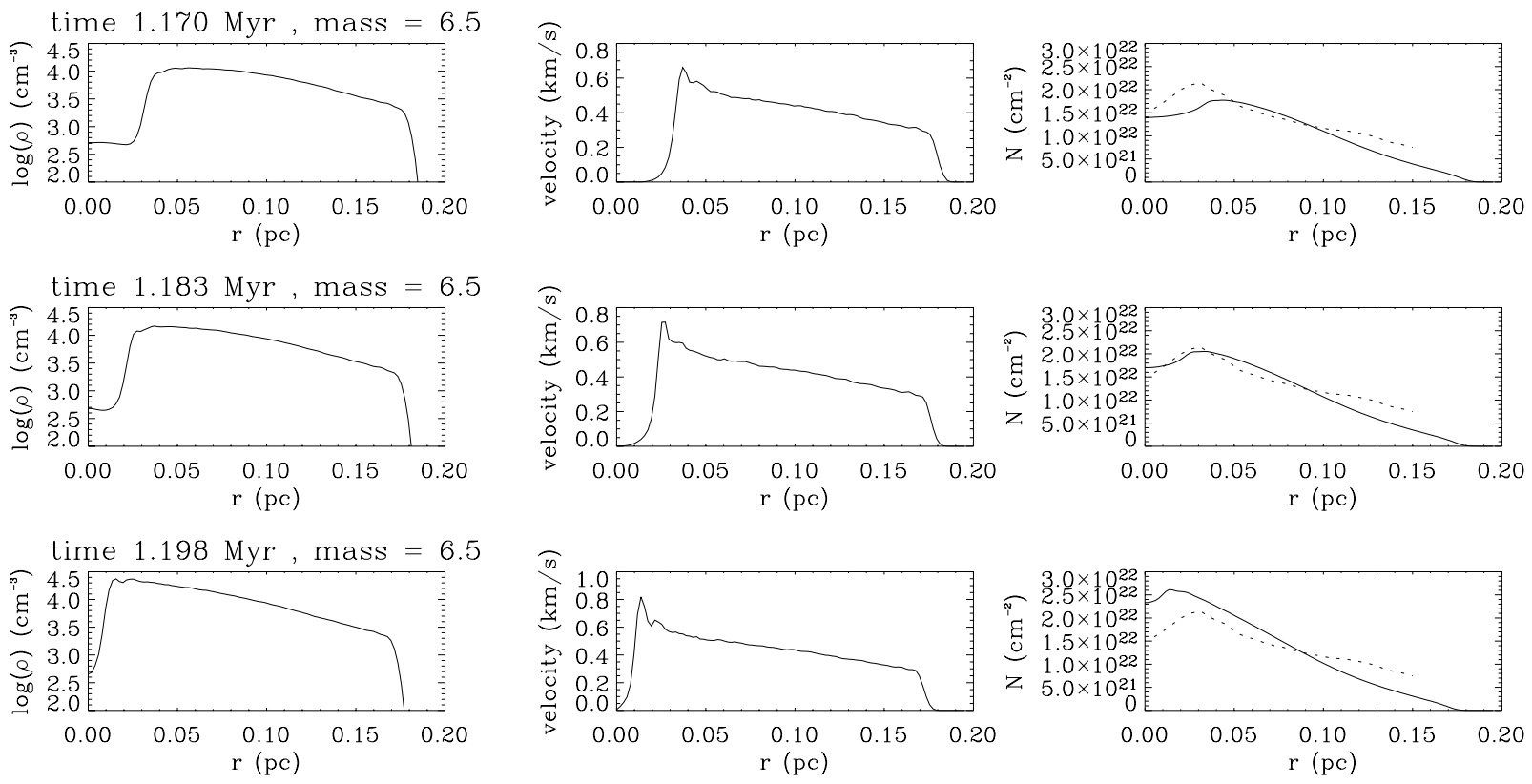

Fig. 1. The lefthand column shows volume-density profiles, $n(r)$ (where $n$ is in $\mathrm{H}_{2} \mathrm{~cm}^{-3}$ and $r$ is in pc. The middle column sows radial velocity profiles, $u(r)$ (where $u$ is in $\mathrm{km} \mathrm{s}^{-1}$ and $r$ is in pc). The righthand column shows column-density profiles $N(b)\left(\right.$ where $N$ is is $\mathrm{H}_{2} \mathrm{~cm}^{-2}$ and $b$ is in pc. The simulation results are represented by solid lines, and the column-density profile derived from the observed extinction is represented by a dashed line. The top row is at time $t=1.170 \mathrm{Myr}$; the middle row is at time $t=1.183 \mathrm{Myr}$; and the bottom row is at time $t=1.198 \mathrm{Myr}$.

deep - approximately tenfold - drop in the volume density $n$ at the centre to produce a $\sim 30 \%$ dip in the column-density $N$; it is very unlikely that the subsonic velocities involved in an oscillating core could reproduce this feature.

It is worth stressing that our model predicts a column density in the external part of the cloud $(x \geq 0.1 \mathrm{pc})$ which is roughly two times lower than the observed column-density. This discrepancy could be due to unrelated gas along the line of sight, or additional gas which has been accreted by the core.

Lada et al. (2004) also report a single line profile for the ${ }^{12} \mathrm{C}^{18} \mathrm{O}(J=2 \rightarrow 1)$ transition from a line of sight which is displaced from the notional centre of symmetry of the core by $\Delta \delta=68.5^{\prime \prime}$ and $\Delta \alpha=69^{\prime \prime}$, giving a net angular displacement of $\sim 100^{\prime \prime}$. At a distance of $D=150 \mathrm{pc}$, this corresponds to an impact parameter $b \sim 0.08 \mathrm{pc}$. We can therefore predict the line profile expected from the simulation, using

$I_{v}=\int j\left(n(r), T_{0}\right)\left\{\frac{\mathrm{d} v^{\prime}}{\mathrm{d} s}\right\}^{-1} \phi\left(v^{\prime}-v\right) \mathrm{d} v^{\prime}$,

where $v$ is the radial velocity (i.e. frequency) observed, $j\left(n(r), T_{0}\right)$ is the integrated volume emissivity in the line, $s$ is distance along the line of sight (measured from the tangent point), $r(s)=\left(b^{2}+s^{2}\right)^{1 / 2}, v^{\prime}(s)=u(r(s)) s / r(s)$ is the inflow velocity projected along the line of sight, and $\phi\left(v^{\prime}-v\right)$ is the profile function (reflecting thermal, turbulent and natural broadening of the line).

Figure 2 shows emission line profiles for ${ }^{12} \mathrm{C}^{18} \mathrm{O}(\mathrm{J}=$ $2 \rightarrow 1$ ) on lines of sight with impact parameters $b=0$, $0.04,0.08$ and $0.12 \mathrm{pc}$. The simulated line profile at $b=0.08 \mathrm{pc}$ matches the width of the broad line component observed by Lada et al. (2004) quite well. We concur with their interpretation of the narrow line component as due to a small-scale
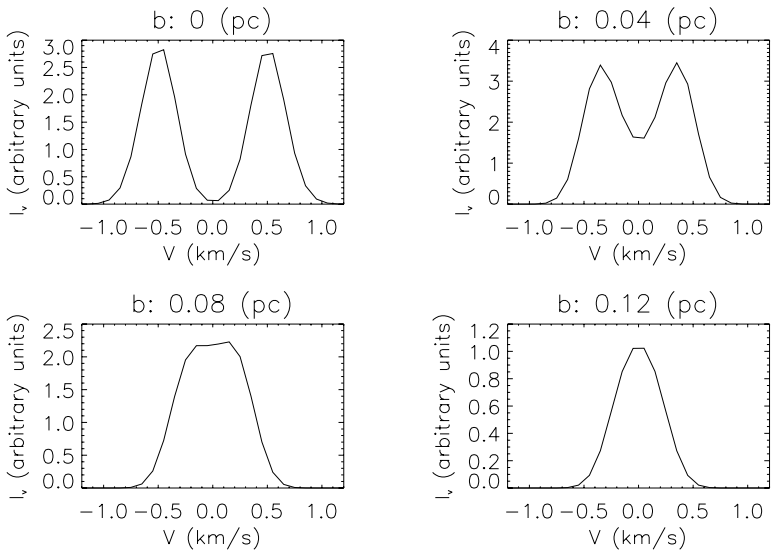

Fig. 2. ${ }^{12} \mathrm{C}^{18} \mathrm{O}(J=2 \rightarrow 1)$ line profiles at time $t=1.183 \mathrm{Myr}$, from lines of sight at different impact parameters $b$, relative to the centre of the simulated core. The impact parameters are $b=$ $0,0.04,0.08$ and $0.12 \mathrm{pc}$.

turbulent fluctuation, and we would not expect the present model to reproduce this. From the profiles presented in Fig. 2, it should be straightforward to test the model proposed here by measuring ${ }^{12} \mathrm{C}^{18} \mathrm{O}(J=2 \rightarrow 1)$ line profiles at different positions within the G2 core. Positions within $\sim 50^{\prime \prime}$ of the centre should present double-peaked line profiles, due to the receding and approaching sides of the compression wave.

\subsection{Effect of turbulence}

Here we present the results of three simulations in which the initial conditions are the same as in Simulation 1, except that a turbulent velocity field is superimposed at the outset. The 

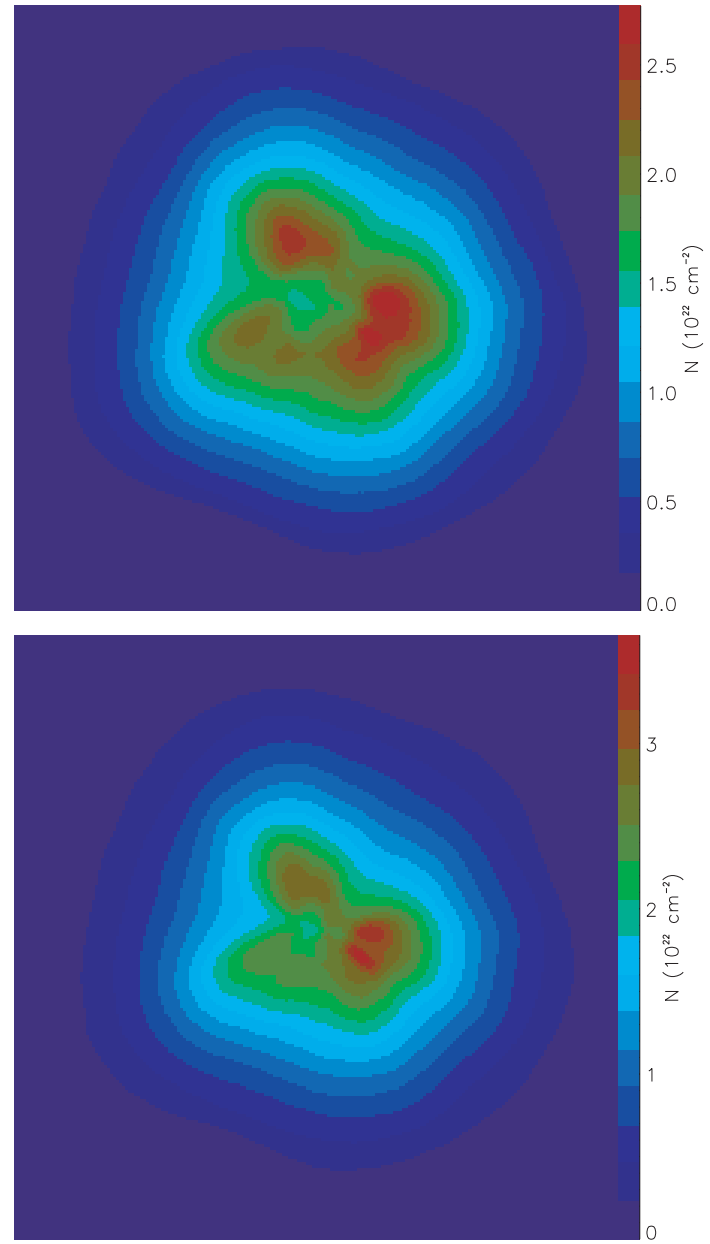

Fig. 3. Column density maps from simulations with initial turbulence. Parameters are: $\alpha_{\text {TURB }}=0.01, n=5 / 3$. Top panel is for time equal to $1.17 \mathrm{Myr}$ whereas second panel is for $1.18 \mathrm{Myr}$.

figures all show column density maps. The different turbulence parameter combinations are given in Table 1 . We note that the levels of turbulence invoked are very low $\alpha_{\mathrm{TURB}}=$ 0.01 and 0.02 . The turbulent power spectra have exponents $n=5 / 3$ (corresponding to Kolmogorov) and 0 (i.e. more power on small scales).

Figure 3 displays the column densities at two times steps close to $t=1.18 \mathrm{Myr}$ which matches best the observation. It shows that even with $\alpha_{\mathrm{TURB}}=0.01$ and $n=5 / 3$ (simulation 2) the ring is significantly distorted. It is still roundish but is fragmented in denser clumps. We note that this is actually the case for the ring observed by Lada et al.

Figure 4 shows that with $\alpha_{\text {TURB }}=0.02$ and $n=5 / 3$ (simulation 3 ) the ring is lost rapidly and completely. The gas rapidly concentrates in one single dense clump.

The only way to retrieve the ring with $\alpha_{\mathrm{TURB}}=0.02$ is to concentrate turbulent energy at short wavelengths by decreasing $n$. This is illustrated in Fig. 5, where $\alpha_{\mathrm{TURB}}=0.02$ and $n=0$ (simulation 4 ), and the ring stays almost axisymmetric during a longer amount of time. However, such a flat power spectrum is unrealistic since usually Kolmogorov-like power spectra are found in observations and in numerical simulations. We note that for large values of $\alpha_{\text {TURB }}(\geq 5 \%)$, the ring is destroyed even with $n=0$.


Fig. 4. Same as Fig. 3 for $\alpha_{\text {TURB }}=0.02, n=5 / 3$. Top panel is for time equal to $1.17 \mathrm{Myr}$ whereas second panel is for $1.185 \mathrm{Myr}$.

We conclude that, within the scope of our model, the ringlike structure observed by Lada et al. (2005) requires the initial core to have a very low level of turbulence. This suggests that the initial core has been very settled before it was subjected to an increase in external pressure, and consequently there was enough time for the turbulence to decay almost completely.

\section{Discussion and conclusions}

We have modelled Tapia's Globule G2 in the Coalsack as a core which is responding to a rapid increase in external pressure. The resulting compression wave has not yet reached the centre, and therefore there is a central rarefaction which manifests itself as a dip in the extinction towards the centre, as observed by Lada et al. (2004). This model requires that the core was already in existence as a coherent entity with relatively low mean density, $\bar{n}_{\text {INIT }} \sim 0.4 \times 10^{3} \mathrm{~cm}^{-3}$, and large extent, $r_{\text {INIT }} \sim 0.4 \mathrm{pc}$, and that the external pressure acting on it increased by a factor $\sim 10$ (from $\sim 0.16 \times 10^{4} k_{\mathrm{B}} \mathrm{cm}^{-3} \mathrm{~K}$ to $\sim 0.16 \times 10^{5} k_{\mathrm{B}} \mathrm{cm}^{-3} \mathrm{~K}$ ) in a small fraction of a soundcrossing time, $t_{\mathrm{SC}} / 30 \sim 0.07 \mathrm{Myr}$. The advantages of such external triggers for synchronising the formation of stars in clusters and generating high multiplicity have been emphasised by Whitworth et al. (1996). 

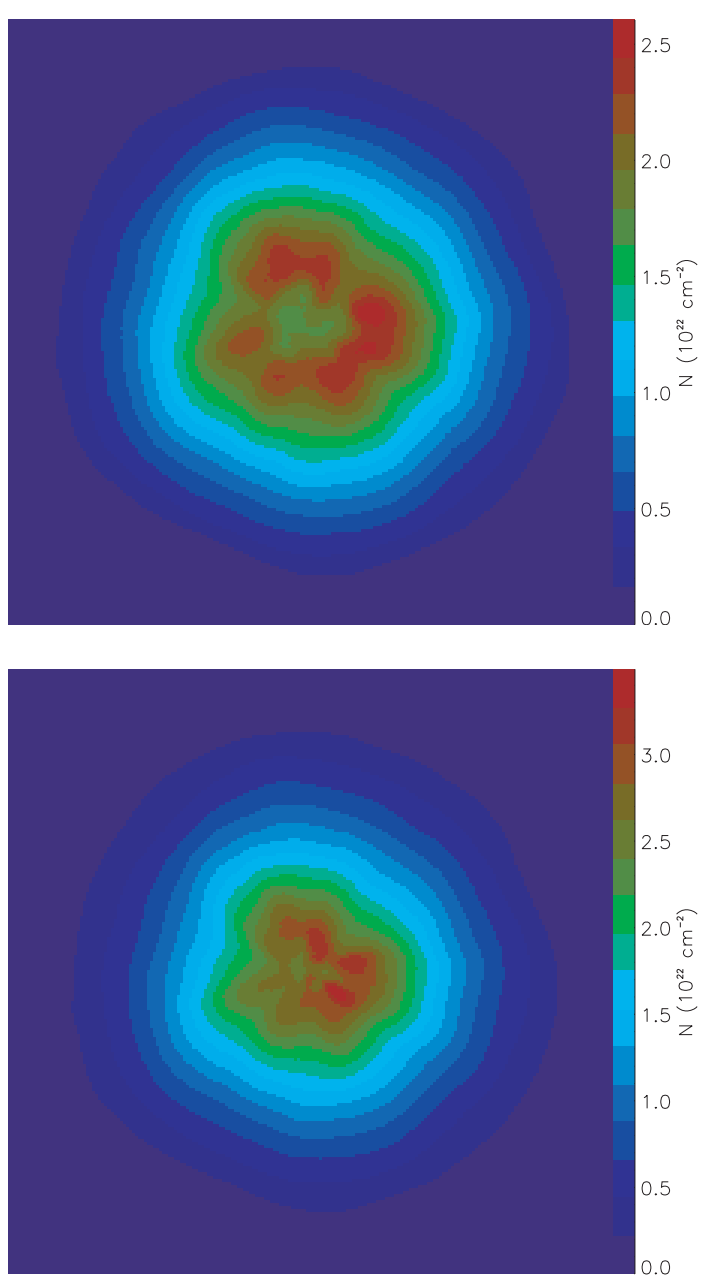

Fig. 5. Same as Fig. 3 for $\alpha_{\mathrm{TURB}}=0.02, n=0$. Top panel is for time equal to $1.175 \mathrm{Myr}$ whereas second panel is for $1.20 \mathrm{Myr}$.

One possibility is that the Coalsack has been involved in, or even created by, a collision between two large-scale turbulent flows. To deliver the required large pressure increase in the required short time requires a flow with minimum density $n_{\mathrm{MIN}} \sim 2 \mathrm{~cm}^{-3}$ and minimum speed $v_{\mathrm{MIN}} \sim 6 \mathrm{~km} \mathrm{~s}^{-1}$. Neither requirement is extreme. Indeed the models of Audit \& Hennebelle (2005) show that pressure increases of the magnitude invoked by our model are very common in the atomic gas from which molecular complexes like the Coalsack are presumed to form.

A second possibility is that the core has fallen into a denser environment and been compressed by the increased ambient pressure. The local cloud within which G2 resides is Region II in the scheme of Nyman et al. (1989); see also Kato et al. (1999). This cloud has mass $M \sim 400 M_{\odot}$ and cross-sectional area $A \sim 9 \mathrm{pc}^{2}$, so its mean surface density is $\bar{\Sigma} \sim M / A \sim$ $0.01 \mathrm{~g} \mathrm{~cm}^{-2}$, and therefore, assuming that it is self-gravitating, its central pressure must be $P_{\mathrm{C}} \sim G \bar{\Sigma}^{2} \sim 0.5 \times 10^{5} k_{\mathrm{B}} \mathrm{cm}^{-3} \mathrm{~K}$. It follows that the core only needs to travel about one third of the cloud radius, i.e. $\sim 0.5 \mathrm{pc}$, to experience the required increase in external pressure, and it can do this in $t_{\mathrm{SC}} / 30 \sim 0.07 \mathrm{Myr}$ by travelling at $\sim 7 \mathrm{~km} \mathrm{~s}^{-1}$, which is a typical bulk velocity for a cool cloud in the local interstellar medium. Since the measured radial velocity of $\mathrm{G} 2$ is $\sim-6 \mathrm{~km} \mathrm{~s}^{-1}$, and the measured radial velocity of the Coalsack is $\sim-5$ to $0 \mathrm{~km} \mathrm{~s}^{-1}$ (Nyman et al. 1989), the relative radial velocity is in the range $\sim 1$ to $5 \mathrm{~km} \mathrm{~s}^{-1}$. Thus if the net velocity of $\mathrm{G} 2$ relative to the Coalsack is $\sim 7 \mathrm{~km} \mathrm{~s}^{-1}$, the chance of its radial component being in this range is $\sim 71 \%$. Given that we have only one case, we cannot take statistical arguments any further, but this at least illustrates that the model is not based on a highly unlikely coincidence. We note that this estimate is based on the assumption that the main effect of the globule falling into a denser region is a pressure increase. However other processes like accretion of more material into the original globule could certainly have a significant impact and promote the formation of a core out of equilibrium.

We have also shown that within the scope of our model the initial level of turbulence in the core (before $t=0$ ) must have been very low $\left(\alpha_{\mathrm{TURB}} \leq 5 \%\right)$ in order for the ring-like structure to develop in a coherent manner. Turbulence promotes fragmentation of the ringlike structure, and generates dense clumps resembling those observed by Lada et al. in the dusty ring in the Coalsack.

However we note that unlike in our simulations, in the observations these overdensities seem to protrude from the ring. We do not know at this stage the reason of this. It may be due to the setting up of turbulent fluctuations with random phases or to incomplete treatment of the physics of the core, since we have ignored the effects of magnetic fields and large-scale anisotropy. Our numerical resolution could also be insufficient to describe accurately such small scale structures.

We reiterate that we have not performed an extensive parameter search to obtain a best-fit model. The observational constraints (the azimuthally averaged, radial extinction profile; and a single, off-centre, approximately pencil-beam, lineprofile) do not justify detailed modelling, so we are here only attempting a feasibility study. The next step in testing the model should be to perform further line observations to test the kinematic predictions of the model (see Fig. 2). We hope to obtain these observations soon. Another line of investigation which should be pursued is to explore numerically the departures from spherical symmetry which can be expected because the pressure increase acting on the core is likely to be anisotropic.

In the meantime, it is appropriate to contrast the predictions of our model with those of the magnetic model developed by Li \& Nakamura (2002).

(i) The magnetic model requires that the core be viewed from close to the axis of symmetry. In contrast, our model can be viewed from any angle, since it is spherically symmetric.

(ii) The magnetic model requires the magnetic field to be mainly along the line of sight. Lada et al. (2004) show that the field component in the plane of the sky (estimated from the dispersion in polarisation angles of background stars, using the Chandraskhar-Fermi equation) is rather close to the equipartition value, and they point out that this rather large field component in the plane of the sky is hard to reconcile with the idea that the main component is along the line of sight. 
(iii) G2 is located towards the edge of the cloud complex, so it is difficult to see how it can evolve quasistatically in the ordered way described by Li \& Nakamura (2002). This presumably requires the field to be anchored in some largerscale structure, but no such structure is evident on the CO maps of Nyman et al. (1989) and Kato et al. (1999).

The model proposed here falls naturally within the category "spherical geometry: a core in transition" defined by Lada et al. (2004). The question then arises as to whether the core will eventually settle into an equilibrium state (a Bonnor-Ebert sphere) or whether it will continue contracting to form a star (or stars) at the centre. Our simulation shows that when the compression wave converges on the centre of the core it creates a primary protostar (cf. Hennebelle et al. 2003, 2004). This is expected to happen in about 40000 years.

Acknowledgements. We thank Maryvonne Gerin for drawing the Lada et al. paper to our attention, and Charlie Lada for useful discussions. This paper was written while APW was a visitor at the École Normale Supérieure, and he is very grateful for the support and hospitality he received during his visit. SPG is supported by a UKAFF Fellowship. We thank Ian Bonnell, the referee, for his comments which have significantly improved the original manuscript.

\section{References}

Alves, J., Lada, C. J., Lada, E. A., Kenyon, S. J., \& Phelps, R. 1998, ApJ, 506, 292

Alves, J., Lada, C. J., \& Lada, E. A. 2001, Nature, 409, 159
André, Ph., Ward-Thompson, D., \& Barsony, M. 2000, in Protostars and Planets IV, ed. V. Mannings, A. P. Boss, \& S. S. Russell (Tucson: Univ. Arizona Press), 59

Audit, E., \& Hennebelle, P. 2005, A\&A, 433, 1

Cambresy, L. 1999, A\&A, 345, 965

Eaton, N., Rolph, C. D., Scarrott, S. M., \& Wolstencroft, R. D. 1990, MN, 244, 527

Goodwin, S. P., Whitworth, A. P., \& Ward-Thompson, D. 2004, A\&A, 414, 633

Harvey, D. W. A., Wilner, D., Alves, J., et al. 2001, ApJ, 563, 903

Hennebelle, P. 2003, A\&A, 411, 9

Hennebelle, P., Whitworth, A. P., Gladwin, P. P., \& André, Ph. 2003, $\mathrm{MN}, 340,870$

Hennebelle, P., Whitworth, A. P., Cha, S.-H., \& Goodwin, S. P. 2004, MN, 348, 687

Kato, S., Mizuno, N., Asayama, S.-I., et al. 1999, PASJ, 51, 883

Lada, C. J., Huard, T. L., Crews, L. J., \& Alves, J. F. 2004, ApJ, 610, 303

Li, Z.-Y., \& Nakamura, F. 2002, ApJ, 578, 256

Matsumoto, T., \& Hanawa, T. 2003, ApJ, 595, 913

Nyman, L.-Å., Bronfman, L., \& Thaddeus, P. 1989, A\&A, 216, 185

Reipurth, B. 1981, A\&ASS, 44, 379

Rodgers, A. W. 1960, MN, 120, 163

Schwartz, R. D. 1977, ApJSS, 35, 161

Tapia, S. 1973, in Interstellar Dust and Related Topics, ed. J. M. Greenberg, \& H. C. van de Hulst (Dordrecht: Reidel), IAU Symp., 52,43

Weaver, W. B. 1973, ApJ, 184, 881

Weaver, W. B. 1974a, ApJ, 189, 81

Weaver, W. B. 1974b, ApJ, 189, 263

Whitworth, A. P., Bhattal, A. S., Francis, N., \& Watkins, S. J. 1996, MNRAS, 283, 1061 